\title{
Transmission concomitante de trypanosomose humaine et animale : le foyer de Mandoul au Tchad
}

\author{
Peka Mallaye ${ }^{1}$ L. Kohagne Tongué ${ }^{2,3 *}$ N. Ndeledje ${ }^{4}$ \\ F.J. Louis ${ }^{5}$ H. Mahamat Hassane ${ }^{3}$
}

\section{Mots-clés}

Maladie de I'homme - Maladie des animaux - Trypanosomose africaine Glossinidae - Stomoxyinae Tabanidae - Epidémie - Tchad.

\begin{abstract}
Résumé
La trypanosomose est une maladie qui affecte à la fois l'homme et les animaux. Elle est provoquée par Trypanosoma sp. et cycliquement transmise par un vecteur, la glossine. Bien que cette maladie soit essentiellement endémique dans l'aire de distribution de son vecteur, les zones endémiques qui présentent une transmission active des deux types de la maladie ont rarement été décrites. Dans la présente étude, des enquêtes épidémiologique et entomologique ont été menées, puis les échantillons obtenus ont été analysés par la technique d'amplification en chaîne par polymérase (PCR). Au total, 13410 personnes ont été examinées et 132 cas diagnostiqués. L'examen de 144 bovins par PCR a révélé l'infection de 33 d'entre eux, soit par Trypanosoma brucei (39 p. 100 des infections), soit par T. vivax (55 p. 100 des infections), soit par une coïnfection (deux animaux). Trois familles d'insectes (Glossinidae, Stomoxyinae et Tabanidae) ont été capturées à des densités variables. Glossina fuscipes fuscipes a été capturée uniquement dans la partie sud du foyer et la plus forte densité apparente (DAP $=0,56$ glossine/piège/jour) a été observée dans la forêt galerie bordant les villages où a été diagnostiqué le plus grand nombre de malades. Les Tabanidae ont été présentes dans toutes les zones prospectées mais la plus forte densité (DAP $=15,55$ tabanidés/piège/jour) a été observée dans la partie nord du foyer. Les stomoxes ont été absents de la zone prospectée la plus éloignée de la rivière. L'identification des trypanosomes chez I'homme et le bétail, et la présence du vecteur cyclique et des vecteurs mécaniques potentiels ont confirmé l'endémie de trypanosomose animale et humaine dans ce foyer. Seule une stratégie globale d'élimination permettrait de la contrôler durablement.
\end{abstract}

La trypanosomose est une maladie parasitaire provoquée par un protozoaire flagellé sanguicole exoerythrocytaire appelé trypanosome, du genre Trypanosoma sp. (Kinetoplastida : Trypanosomatidea). Diverses espèces et sous-espèces de trypanosomes sont

\footnotetext{
1. Programme de lutte contre la trypanosomiase humaine africaine du Tchad, Moundou, Tchad.

2. Association pour la promotion de la lutte contre les parasitoses, Yaoundé, Cameroun.

3. Pan African Tsetse and Trypanosomiasis Eradication Campaign, Addis Ababa, Ethiopia.

4. Ministère de la Recherche, N’Djaména, Tchad.

5. Association against trypanosomiasis in Africa, Lavans-Saint Lupicin, France.

* Auteur pour la correspondance

Tél. : +23722103849

E-mail : lisetteappmv@yahoo.fr; aplp_contact@yahoo.fr
}

responsables de cette maladie chez les vertébrés : chez l'homme, on parle de trypanosomiase ou trypanosomose humaine africaine (THA), et chez les animaux on parle de trypanosomose animale africaine (TAA). La trypanosomose de façon générale (THA, TAA) est une maladie à transmission vectorielle dont le vecteur cyclique est la glossine ou mouche tsé-tsé, du genre Glossina (Diptera : Glossinidae).

La forme humaine sévit exclusivement en Afrique dans l'aire de distribution des glossines. Cependant, la présence de glossines n'induit pas forcément celle de la THA. La maladie est présente essentiellement dans des zones géographiques bien circonscrites appelées foyers où cohabitent le vecteur, le parasite et l'homme. Environ 250 foyers (6) ont été répertoriés à ce jour mais tous ne présentent pas la même intensité de transmission. La transmission de la maladie varie d'un pays à l'autre et, au sein d'un même pays, d'un foyer à l'autre (6), notamment en ce qui concerne la forme due à T. brucei gambiense. Certains foyers ont une intensité de transmission élevée. Ce 
sont les foyers rapportant une moyenne annuelle d'au moins un nouveau cas pour 1000 habitants. D'autres ont une intensité de transmission modérée (au moins un nouveau cas pour 10000 habitants mais moins d'un nouveau cas pour 1000 habitants), d'autres encore une faible intensité de transmission (un nouveau cas pour 1000000 habitants mais moins d'un nouveau cas pour 10000 habitants (39). La transmission de la trypanosomose est en effet intimement liée à la fréquence du contact entre l'hôte et le vecteur.

Dans la forme animale, cette transmission active peut être renforcée par une transmission mécanique assurée par des Stomoxyinae, des Tabanidae et quelques Hippoboscidae (11) qui maintiennent l'incidence de la maladie, même dans des aires dépourvues de glossines (41). Alors que l'intensité de la transmission cyclique de la trypanosomose n'est pas absolument liée à la densité du vecteur cyclique qu'est la glossine, celle de la transmission mécanique est étroitement liée à la densité des vecteurs, à la proximité et à l'abondance numérique du cheptel dans une aire donnée, et à la parasitémie (11).

Bien que la trypanosomose soit endémique en Afrique, il a rarement été rapporté des aires de transmission active où cohabitent la forme humaine et la forme animale de la maladie. Nous décrivons ici la situation épidémio-entomologique du foyer de Mandoul au Tchad.

\section{MATERIEL ET METHODES}

\section{Zone d'étude}

Anciennement appelé foyer de Bodo ( $8^{\circ} 17^{\prime} 08^{\prime}$ ' de lat. $\mathrm{N}$ et $17^{\circ} 08^{\prime} 22$ " de long. E), le foyer de THA de Mandoul est localisé au sud du Tchad, à $50 \mathrm{~km}$ de la ville de Doba et à $150 \mathrm{~km}$ de Moundou, la capitale économique du Tchad. Il doit son nom à sa position à cheval sur la rivière semi-permanente dénommée Mandoul qui parcourt le sud du Tchad pour se jeter dans le Logone.

C'est en 1928 que, pour la première fois, une épidémie de THA fut rapportée dans le canton de Bodo (5). Après des efforts continus de lutte dans tout le pays et particulièrement dans cette localité, la THA disparut quasiment de Bodo et même du Tchad dans les années 1960 (32). La maladie redevint active de façon officielle après plus de quarante années de silence en 1993, avec 201 malades diagnostiqués (prévalence de 4,61 p. 100) (F. Boyer, rapport d'évaluation épidémiologique, non publié). Ce n'est pourtant qu'à partir de 2002 que des actions de lutte coordonnées et efficaces ont été mises en œuvre par un programme de lutte restructuré, avec l'appui de l'Organisation mondiale de la santé (OMS) et de l'Organisation de coordination pour la lutte contre les endémies en Afrique centrale (Oceac). Le foyer de Bodo (devenu Mandoul) est alors estimé couvrir une superficie de $15 \mathrm{~km}$ environ de large par $20 \mathrm{~km}$ de long (22). C'est une zone en perpétuelle expansion démographique et il est assez difficile d'y évaluer précisément le nombre de villages. Au moment de sa délimitation, il comptait 45 villages et campements : 30 sur la rive gauche de la rivière, 14 sur la rive droite, et un village, Solangar, sur une île au milieu de la rivière (23). Aujourd'hui, on estime à plus de 60 le nombre de villages que compte ce foyer avec une moyenne de 30 de chaque côté de la rivière, le village Solangar ayant, lui, disparu (figure 1). Le climat y est de type soudano-guinéen avec deux saisons : une saison des pluies de mai à septembre et une saison sèche d'octobre à avril. Les températures varient entre 22 et $38^{\circ} \mathrm{C}$ et la pluviométrie moyenne annuelle est de $1000 \mathrm{~mm}(25)$.

Le paysage est marqué par un seul type de végétation, la forêt galerie qui longe la rivière Mandoul. C'est une zone densément

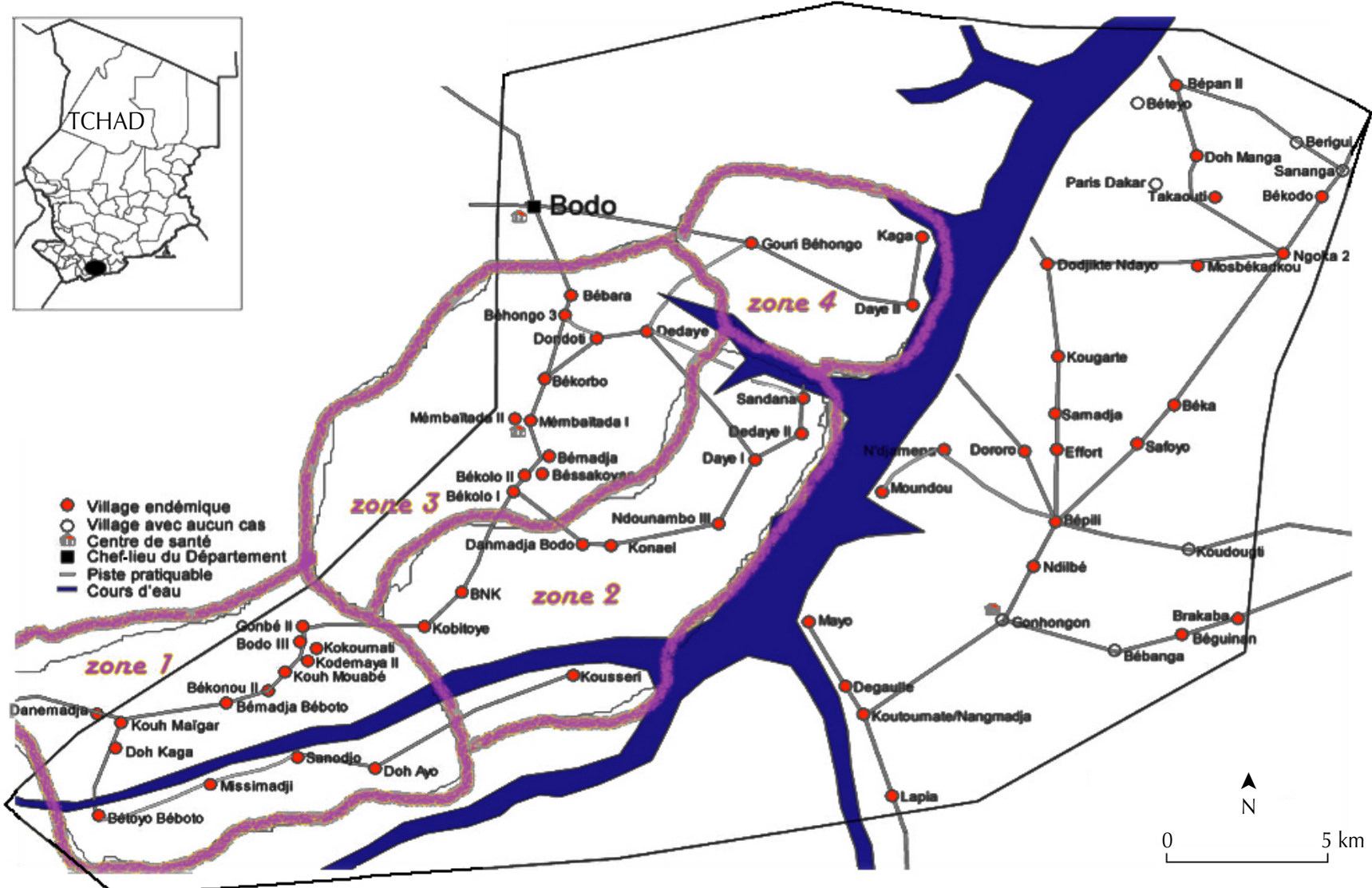

Figure 1 : les quatre zones prospectées du foyer de Mandoul au Tchad. Les villages endémiques et indemnes sont ceux identifiés en 2011, lors de l'élaboration de la carte. 
peuplée, 9 habitants $/ \mathrm{km}^{2}$ en 2004, contre 0,2 habitant $/ \mathrm{km}^{2}$ dans le nord du Tchad (enquête Aquastat 2005 non publiée), en raison de la fertilité des sols proches de la rivière. Ainsi, de vastes champs de coton, de mil et de sésame surplombent la forêt galerie au-delà de laquelle sont construites les cases des villages. Cette population, constituée en majorité par l'ethnie Sara, pratique également un élevage extensif de bovins, d'ovins, de caprins et de porcins. En saison sèche, la rivière Mandoul asséchée dans sa partie nord offre une prairie à de nombreux éleveurs Bororos en transhumance.

L'enquête épidémiologique s'est inscrite au sein d'une campagne conjointe de prospection médicale et vétérinaire conduite au mois de novembre 2012.

\section{Prospection médicale}

La campagne de dépistage actif a eu lieu du 9 au 23 novembre 2012 et a concerné 42 villages : 37 situés sur la rive gauche, dont 32 déjà répertoriés en 2011, et 5 villages nouvellement créés, puis 5 villages situés entre les deux premiers bras de la rivière, côté sud. Elle s'est déroulée après une sensibilisation des autorités et des populations locales par l'équipe médicale. Tous les villages retenus ont été prospectés et la population, estimée à partir des prospections antérieures conduites annuellement depuis 2009, comportait 16685 personnes.

La chaîne de dépistage a été organisée suivant le schéma décisionnel classique (figure 2) établi par l'OMS (40) mais modifié (19) afin de permettre d'examiner jusqu'à 1500 personnes par jour. Ces modifications portent essentiellement sur le dédoublement des postes de secrétariat et de sérologie (card agglutination test for trypanosomosis [CATT] [24] sur sang total et CATT titration) que l'on place dans des villages voisins (si leur population est limitée) ou à deux extrémités d'un même village de population importante. Les anciens malades ont été ôtés de la chaîne (après le test CATT sur sang total suivi d'un questionnaire) dès leur identification. Les sujets positifs au CATT sur sérum à la titration 1/8 (négatifs à la titration 1/16) ont été libérés et considérés comme des suspects sérologiques. Seule la centrifugation en tubes capillaires a été utilisée pour l'examen parasitologique.

Tous les sujets positifs au moins à la titration 1/16 mais négatifs à la parasitologie (aucun trypanosome détecté) ont été reconnus

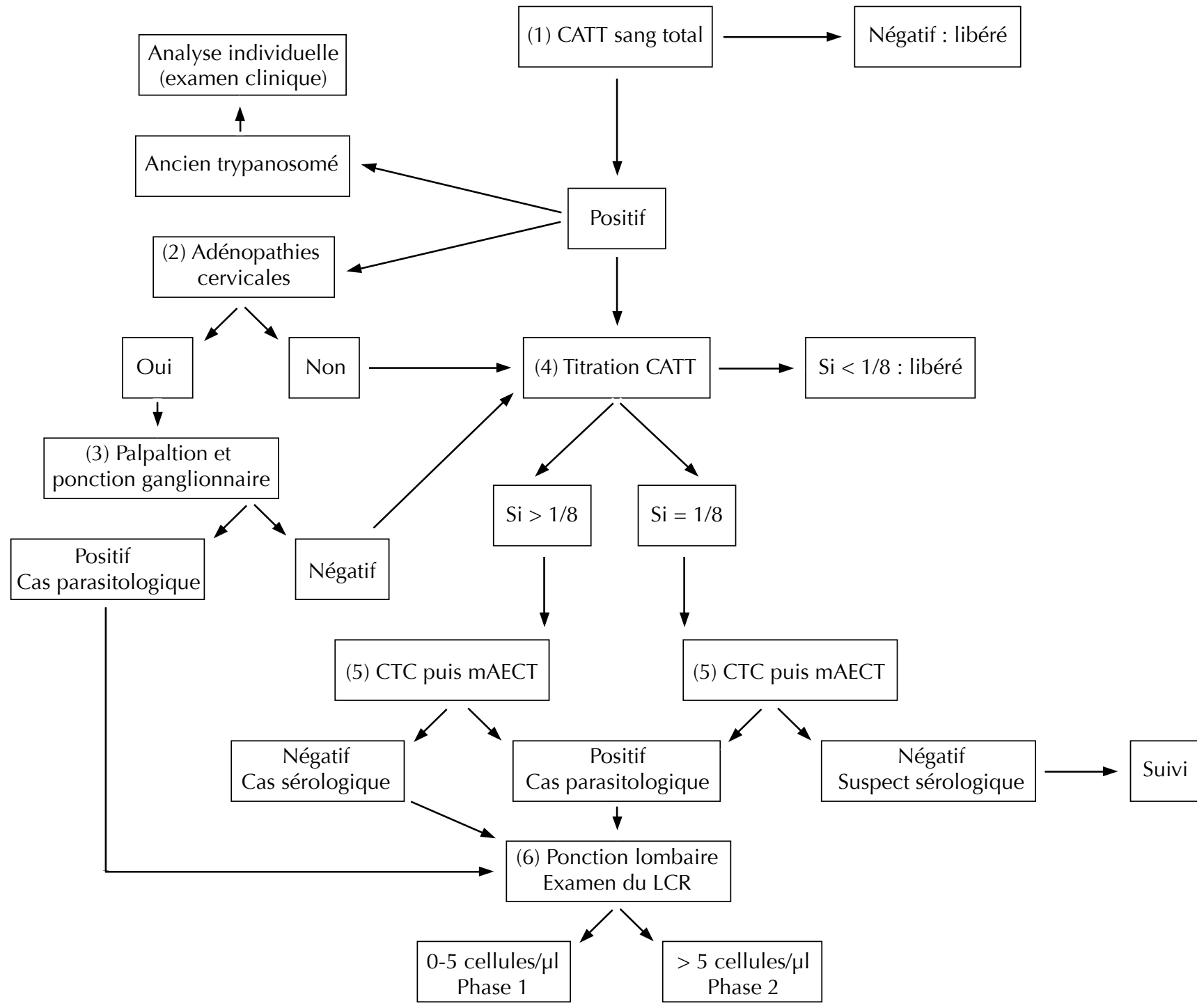

Figure 2 : chaîne de dépistage classique de la THA utilisée par le Programme national de lutte contre la trypanosomiase humaine africaine (PNLTHA) du Tchad

CATT : card agglutination test for trypanosomosis ; $C A T T_{W B}=C A T T$ sur sang total ; CTC = centrifugation en tube capillaire ; mAECT : minicolonne échangeuse d'ions 
comme des cas sérologiques. Les cas sérologiques et parasitologiques (individus chez qui le trypanosome a été détecté) ont ensuite été mis sous traitement : pentamidine pour les malades en première phase, combinaison thérapeutique de nifurtimox et d'éflornithine (NECT) pour ceux en deuxième phase. La phase a été définie par la cytorachie : inférieure ou égale à cinq leucocytes par microlitre pour la phase lymphatico-sanguine (phase 1), supérieure à cinq leucocytes pour la phase méningo-encéphalitique (phase 2).

\section{Prospection vétérinaire}

La prospection vétérinaire s'est déroulée du 15 au 30 novembre 2012 et n'a concerné que le bétail sédentaire des villages de la rive gauche, c'est-à-dire les villages ayant bénéficié de la prospection médicale. Avant la phase de prospection, les propriétaires de bovins ayant accepté de prendre part à l'enquête ont été conviés à répondre à un questionnaire regroupant entre autres des informations sur le nombre de têtes, la race, le poids, le sexe et l'âge des animaux composant leur cheptel respectif. Ces données ont ensuite été regroupées par sexe et par âge (stratification). Pour des raisons d'échantillonnage, la zone de l'étude a été subdivisée en quatre parties pendant la prospection : zone 1 , zone 2 , zone 3 et zone 4 (figure 1). Dans chacune des zones, les bovins ont été sélectionnés selon un échantillonnage stratifié (par âge et par sexe) aléatoire. Ils ont ensuite été examinés et quelques signes cliniques (par exemple larmoiement, amaigrissement, fièvre, poil piqué, présence ou non d'hypertrophie ganglionnaire) ont été enregistrés.

Les prélèvements ont été effectués à la veine jugulaire dans des tubes héparinés de cinq millilitres. L'observation microscopique du sang entre lame et lamelle, réalisée dans les deux heures ayant suivi le prélèvement, a constitué le seul examen parasitologique effectué sur le terrain. La mesure de l'hématocrite a complété cette phase d'analyse. Le plasma a été récolté après centrifugation et imprégné sur papier Whatman ${ }^{\circledR}$ puis conservé pour l'identification ultérieure des espèces de trypanosomes.

\section{Enquête entomologique}

L'enquête entomologique s'est également déroulée du 15 au 30 novembre 2012, dans les quatre zones de la rive gauche du foyer identifiées lors de l'enquête épidémiologique vétérinaire. Pour des raisons de logistique, cette enquête n'a couvert qu'un seul bras de la rivière, celui aux abords duquel étaient établis des villages dont certains s'étaient avérés endémiques de THA lors de la prospection médicale. Trois types de pièges ont été utilisés eu égard à la diversité des mouches ciblées : les pièges Vavoua (20) et biconique (7), efficaces surtout pour la capture des glossines, et le piège Nzi (28), efficace pour la capture des stomoxes et des tabanidés. Les sites de piégeage, éloignés les uns des autres de 100 à $150 \mathrm{~m}$, ont été choisis sur la base de leurs caractéristiques environnementales favorables à la survie des glossines. Aucun piège n'a ainsi été posé à la lisière des villages où le couvert végétal était très faible. Dans chacun de ces sites, les trois types de pièges ont été posés à une distance de 2 à $5 \mathrm{~m}$ les uns des autres malgré l'interférence que pourrait occasionner ce rapprochement dans le calcul de la densité des glossines. L'objectif de cette enquête a été en effet de montrer, outre le vecteur cyclique, l'existence de vecteurs mécaniques potentiels de TAA dans la zone prospectée. La densité a été estimée par la densité apparente par piège (DAP) calculée ainsi : $\mathrm{DAP}=\frac{\text { Nombre total de mouches capturées }}{\text { Nombre total de pièges } \mathrm{x} \text { nombre de jours de capture }}$
Dans la zone 1 regroupant 13 villages prospectés lors de l'enquête épidémiologique, 16 sites de piégeage ont été retenus et 48 pièges posés ; dans la zone 2 regroupant 10 villages, 10 sites de piégeage ont été retenus et 30 pièges posés ; dans la zone 3 plus éloignée de la rivière et regroupant 11 villages, 4 sites de piégeage ont été retenus et 12 pièges posés; et dans la zone 4 regroupant 3 villages, 9 sites de piégeage ont été retenus et 27 pièges posés. Au total, 117 pièges des trois types ont été utilisés dans 39 sites de piégeage. La base des piquets soutenant les pièges ont été à chaque fois enduite de graisse afin de protéger les mouches emprisonnées dans la cage des fourmis prédatrices. Les pièges ainsi posés étaient laissés en place pendant quatre jours consécutifs et visités deux fois par jour, entre $7 \mathrm{~h}$ et $10 \mathrm{~h}$, puis entre $17 \mathrm{~h}$ et $18 \mathrm{~h} 30$. Les mouches capturées ont été collectées, identifiées sur la base des critères morphologiques publiés par de nombreux auteurs $(4,13,35)$ puis dénombrées. Les glossines fraîches (81 au total) ont été disséquées au niveau du proboscis, de l'intestin et des glandes salivaires en vue de la recherche de l'infection trypanosomienne.

\section{Analyse des échantillons au laboratoire}

Elle n'a concerné que les échantillons sanguins prélevés sur les bovins. Au laboratoire, l'ADN a été extrait avec du Chelex $100^{\circledR}$ de la façon suivante : le papier Whatman ${ }^{\circledR}$ imprégné a été placé dans un tube Eppendorf ${ }^{\circledR}$ contenant $1 \mathrm{ml}$ d'une solution de Chelex $100^{\circledR} 5$ p. $100(\mathrm{w} / \mathrm{v})$, puis chauffé à $55^{\circ} \mathrm{C}$ pendant une heure et à $95{ }^{\circ} \mathrm{C}$ pendant $30 \mathrm{~min}$. Après une centrifugation à $15000 \mathrm{rpm}$ pendant $10 \mathrm{~min}$, le surnageant contenant l'ADN a été collecté et conservé à $-20{ }^{\circ} \mathrm{C}$ pour l'analyse par PCR. Trois couples d'amorces spécifiques aux espèces Trypanosoma (Nanomonas) congolense type savane (30), T. (Trypanozoon) brucei s.l. et $T$. (Duttonela) vivax (26) ont été utilisés.

La réaction d'amplification a été faite dans un volume total de $25 \mu \mathrm{l}$ contenant $1 \mu \mathrm{l}$ d'ADN extrait, $1 \mu \mathrm{l}$ de chacune des amorces testées, 0,25 unité de Taq (Thermos Aquaticus) DNA polymerase et $5 \mu \mathrm{l}$ de tampon PCR enrichi en $45 \mathrm{mM}$ Tris- $\mathrm{HCl}(\mathrm{pH} 8,8)$, $11 \mathrm{mM}$ (NH4)2SO4, 4,5 mM MgCl2, 6,7 mM 2-mercaptoéthanol, 4,4 $\mu \mathrm{M}$ EDTA, $113 \mu \mathrm{g} / \mathrm{ml}$ BSA, $1 \mathrm{mM}$ de chacun des désoxynucléotides phosphate (dTTP, dATP, dCTP et dGTP). L'ADN génomique a été utilisé comme témoin positif et de l'eau stérile comme témoin négatif. Une dénaturation à $94^{\circ} \mathrm{C}$ pendant 4 min a précédé 30 cycles d'amplification comprenant chacun une dénaturation à $95^{\circ} \mathrm{C}$ pendant $1 \mathrm{~min}$, une hybridation à $55^{\circ} \mathrm{C}\left(\mathrm{T}\right.$. brucei) ou $60^{\circ} \mathrm{C}$ (T. vivax, $T$. congolense) pendant $1 \mathrm{~min}$, une élongation à $65^{\circ} \mathrm{C}$ pendant $1 \mathrm{~min}$, puis une élongation finale à $72^{\circ} \mathrm{C}$ pendant $4 \mathrm{~min}$. Les produits d'amplification ont été visualisés sous rayons ultraviolets sur gel d'agarose à 1,5 p. 100 enrichi au SYBR ${ }^{\circledR}$-Safe DNA gel stain.

\section{RESULTATS}

\section{Prospection médicale}

$\mathrm{Au}$ total, 13410 personnes ont été examinées soit un taux de couverture de 80,3 p. 100 ; 132 cas ont été dépistés dont 99 cas parasitologiques (individus porteurs de trypanosomes) et 33 cas sérologiques (individus positifs au CATT titration supérieure ou égale à 1/16). Ces individus (cas sérologiques et parasitologiques) mis sous traitement étaient pour la plupart (96 malades) en phase lymphatico-sanguine. Kobitoye avec 25 malades et Konael avec 10 malades ont été les villages où un nombre élevé de malades ont été diagnostiqués et étaient situés sur la rive gauche de la rivière. Sanodjo avec 10 malades et Doh Ayo avec 10 malades étaient situés entre les deux premiers bras de la rivière. Dans les autres villages, le nombre total de cas diagnostiqués a varié entre 1 et 5 . 
Les suspects sérologiques (individus positifs à la titration CATT égale à $1 / 8$ ) ont été au nombre de 57 . Ces derniers n'ont pas été traités, conformément à l'algorithme de dépistage utilisé.

\section{Prospection vétérinaire}

Dans ce foyer, le système d'élevage était essentiellement de type sédentaire. Au total, 144 bovins dont 77 mâles, tous de race Bororo, ont été examinés dans les quatre zones. L'âge des animaux a varié entre 1 et 12 ans, leur état général était bon pour la plupart (122/144 examinés) et les seuls signes cliniques observés chez 22 animaux ont été le larmoiement et l'hypertrophie ganglionnaire.

L'examen direct a permis de diagnostiquer 12 animaux infectés dont cinq mâles. Huit animaux ont été suspectés (sur la base des mouvements des trypanosomes) d'être infectés par T. brucei et 5 par T. vivax (il y avait un cas suspect d'infection mixte) pour une prévalence apparente globale de 8 p. 100. Parmi ces 12 animaux, 7 avaient un taux d'hématocrite compris entre 25 et 50 p. 100 et 5 un taux d'hématocrite inférieur à 25 p. 100.

L'analyse au laboratoire a concerné 144 échantillons, soit tous les bovins de l'étude. Après analyse par PCR, 33 animaux se sont avérés infectés avec une prévalence variant de 22 à 25 p. 100 par zone prospectée (tableau I). La PCR a permis de confirmer 9 des 12 cas positifs observés entre lame et lamelle. Deux espèces de trypanosomes ont été identifiées : T. brucei (39 p. 100 des infections) et $T$. vivax (55 p. 100), avec 2 cas d'infection mixte à $T$. vivax et $T$. brucei (6 p. 100). T. congolense type savane n'a pas été identifié.

\section{Enquête entomologique}

Trois familles d'insectes vecteurs (cyclique et mécanique) de trypanosomoses ont été identifiés : Glossinidae, Stomoxyinae et Tabanidae. Les trois pièges utilisés ont chacun capturé les trois familles d'insectes ciblées mais l'abondance et la distribution de ces insectes n'a pas été uniforme à l'intérieur du foyer (tableau II).

Seules les Tabanidae ont été présentes dans l'ensemble du foyer à des densités variables dont la plus élevée $(\mathrm{DAP}=15,55 \mathrm{~T} / \mathrm{P} / \mathrm{J})$ a été observée dans la zone 4. Cinq genres ont été identifiés : Tabanus $(\mathrm{DAP}=2,67 \mathrm{~T} / \mathrm{P} / \mathrm{J})$, Chrysops $(\mathrm{DAP}=1,41 \mathrm{C} / \mathrm{P} / \mathrm{J})$, Ancala $(\mathrm{DAP}=0,04 \mathrm{An} / \mathrm{P} / \mathrm{J})$, Atylotus $(\mathrm{DAP}=0,57 \mathrm{~A} / \mathrm{P} / \mathrm{J})$ et Haematopota $(\mathrm{DAP}=0,01 \mathrm{H} / \mathrm{P} / \mathrm{J})$. Aucun stomoxe n'a été capturé dans la zone 3 . Ils ont été capturés en majorité ( $\mathrm{DAP}=4,58 \mathrm{~S} / \mathrm{P} / \mathrm{J})$ dans la zone 4 (tableau III) et leur identification s'est limitée au genre Stomoxys pour de nombreux spécimens, excepté pour l'espèce Stomoxys calcitrans. La plus faible abondance d'insectes a été observée chez les Glossinidae : $\mathrm{DAP}=0,07 \mathrm{G} / \mathrm{P} / \mathrm{J}$ dans la zone 1

\section{Tableau I}

Prévalence apparente de la trypanosomose animale africaine estimée par PCR dans le foyer de Mandoul, Tchad

\begin{tabular}{|c|c|c|c|c|c|c|c|c|}
\hline \multirow[t]{2}{*}{ Zone } & \multirow[t]{2}{*}{$\begin{array}{c}\text { Cheptel estimé } \\
\text { (nb. têtes) }\end{array}$} & \multirow[t]{2}{*}{$\begin{array}{l}\text { Effectif } \\
\text { examiné }\end{array}$} & \multicolumn{4}{|c|}{$\begin{array}{l}\text { Nb. d'animaux } \\
\text { infectés }\end{array}$} & \multirow[t]{2}{*}{ Prévalence (\%) } & \multirow[t]{2}{*}{$\begin{array}{c}\text { Intervalle de } \\
\text { confiance }(95 \%)\end{array}$} \\
\hline & & & Tv & Tb & Tc & Tv-Tb & & \\
\hline I & 150 & 32 & 3 & 3 & 0 & 1 & 22 & [8-36] \\
\hline II & 150 & 41 & 4 & 5 & 0 & 0 & 22 & [9-35] \\
\hline III & 100 & 40 & 7 & 3 & 0 & 0 & 25 & [12-38] \\
\hline IV & 200 & 31 & 4 & 2 & 0 & 1 & 23 & [8-37] \\
\hline
\end{tabular}

PCR : technique d'amplification en chaîne par polymérase

Tv : Trypanosoma vivax; Tb : Trypanosoma brucei s.1.; Tc : Trypanosoma congolense type savane ; Tv-Tb : cö̈nfection T. vivax et T. brucei s.l.

\section{Tableau II}

Nombre d'insectes capturés par zone en fonction du type de piège dans le foyer de Mandoul, Tchad

\begin{tabular}{|c|c|c|c|c|c|c|}
\hline & Vecteurs capturés & $\begin{array}{l}\text { Zone I } \\
48 \mathrm{P} *\end{array}$ & $\begin{array}{c}\text { Zone II } \\
30 \mathrm{P} *\end{array}$ & $\begin{array}{c}\text { Zone III } \\
12 \mathrm{P}^{*}\end{array}$ & $\begin{array}{c}\text { Zone IV } \\
27 \text { P* }\end{array}$ & Total \\
\hline \multirow[t]{3}{*}{ Piège Nzi } & Tabanidae & 153 & 104 & 11 & 802 & 1070 \\
\hline & Stomoxyinae & 8 & 76 & 0 & 197 & 281 \\
\hline & Glossinidae & 2 & 10 & 0 & 0 & 12 \\
\hline \multirow[t]{3}{*}{ Piège biconique } & Tabanidae & 34 & 19 & 7 & 321 & 381 \\
\hline & Stomoxyinae & 3 & 18 & 0 & 181 & 202 \\
\hline & Glossinidae & 6 & 44 & 0 & 0 & 50 \\
\hline \multirow[t]{3}{*}{ Piège Vavoua } & Tabanidae & 132 & 55 & 8 & 556 & 751 \\
\hline & Stomoxyinae & 22 & 8 & 0 & 117 & 147 \\
\hline & Glossinidae & 6 & 13 & 0 & 0 & 19 \\
\hline
\end{tabular}

\footnotetext{
* $\mathrm{P}=$ nombre de pièges posés par zone
} 


\section{Tableau III}

Densité apparente par piège des vecteurs potentiels de trypanosomes capturés dans le foyer de Mandoul, Tchad

\begin{tabular}{|cccc} 
Zone & \multicolumn{3}{c}{ Densité apparente par piège et par jour } \\
\cline { 2 - 4 } & Tabanidae & Stomoxyinae & Glossinidae \\
\hline I & 1,66 & 0,17 & 0,07 \\
II & 1,48 & 0,85 & 0,56 \\
III & 0,55 & 0,00 & 0,00 \\
岕 & 15,55 & 4,58 & 0,00
\end{tabular}

et $\mathrm{DAP}=0,56 \mathrm{G} / \mathrm{P} / \mathrm{J}$ dans la zone 2. Dans les zones 3 et 4 situées en aval de la rivière, aucune glossine n'a été capturée (tableau III). Dans cette famille, seule l'espèce $G$. fuscipes a été identifiée et aucune glossine infectée n'a été observée après dissection.

\section{DISCUSSION}

Le taux de couverture du dépistage actif effectué, c'est-à-dire le rapport de la population examinée sur la population estimée, rend compte de la qualité de la sensibilisation d'une population déjà parfaitement au fait de la maladie et de sa gravité. Avec 132 cas dont 99 confirmés par le diagnostic parasitologique, le foyer de THA de Mandoul est un foyer de transmission élevée. Depuis 2009, au moins un dépistage actif y est conduit par an. Le nombre total de malades notifiés après des dépistages passifs et actifs a été de 510 en 2009, 232 en 2010, et 276 en 2011 (39).

Les cas (parasitologiques et sérologiques) diagnostiqués au cours de notre prospection et lors des enquêtes antérieures étaient des nouveaux cas, au regard de l'arbre décisionnel du dépistage actif mis en place. Cependant, les suspects diagnostiqués n'ont pas été suivis comme il est pourtant recommandé, et ce, pour diverses raisons dont l'insuffisance d'un accompagnement technique (personnels qualifiés, matériels, etc.). Sachant que la première période de l'infection à T. brucei gambiense est marquée par des fluctuations parasitémiques liées au cycle de variation antigénique - élaboration de nouveaux anticorps qui limitent la sensibilité des tests diagnostiques (18), les suspects sérologiques non traités et non suivis de ce foyer pourraient être porteurs du parasite et constituer ainsi un réservoir humain potentiel dans ce foyer où, au moment de notre enquête, aucune lutte antivectorielle n'était mise en œuvre.

Néanmoins, le succès de la régularité du dépistage actif, montré ici par le nombre élevé de personnes diagnostiquées en phase 1, contribue à l'assainissement de ce réservoir humain potentiel de trypanosomes car le diagnostic précoce réduit le risque de transmission de la maladie au sein d'une communauté (8). Toutefois, le dépistage actif ne pourrait à lui seul conduire à l'élimination de la maladie du sommeil d'un foyer à T. $b$. gambiense (39). Les personnes diagnostiquées et traitées sont à nouveau exposées aux piqûres de glossines une fois de retour chez elles, et par conséquent à nouveau exposées au risque de contracter la maladie.

Les villages ayant rapporté un nombre élevé de malades étaient situés dans les zones 1 et 2 où des glossines ont été capturées. Tous les villages de ces deux zones étaient d'ailleurs considérés comme étant à l'épicentre du foyer au moment de sa délimitation (22). Ils sont assez proches de la forêt galerie et densément peuplés (contrairement à ceux de la zone 4), mais la proximité de ces villages de la zone infestée de glossines ne justifie pas à elle seule le grand nombre de malades diagnostiqués. Cette partie de la rivière n'est jamais complètement asséchée. La présence permanente d'un plan d'eau à cet endroit ne favorise pas seulement l'existence d'une hygrométrie relative propice à la survie des glossines, mais aussi l'existence des zones agraires et des pâturages utilisés par les populations.

Sachant que la transmission de la maladie du sommeil est intimement liée à l'intensité du contact entre l'hôte et le vecteur, le nombre élevé de malades serait ainsi lié à la fréquentation régulière par les populations de cette partie du foyer pour leurs activités. En effet, la seule présence d'un point d'eau multiplie au moins par trois le contact entre la glossine et l'homme (21).

Une seule espèce de glossine a été capturée dans la zone prospectée. Pourtant l'espèce G. tachinoides, et les sous-espèces G. fuscipes fuscipes et $G$. morsitans submorsitans signalées il y a longtemps dans le sud du Tchad $(14,17)$ sont encore observées dans la région de Sahr, à un peu plus d'une centaine de kilomètres (31) du foyer de Mandoul. Bien que nos résultats corroborent les observations de certains auteurs (16), il est encore trop tôt, eu égard à la technique d'échantillonnage utilisée et à la superficie du foyer, pour déclarer une probable disparition de G. m. submorsitans du foyer de Mandoul, où cette espèce a été signalée il y a moins d'une vingtaine d'années (10). G. m. submorsitans, fortement inféodée au gros gibier (phacochères, buffles et cobs), a tendance à disparaître dès que les densités humaines augmentent (38), comme c'est le cas dans le foyer de Mandoul.

Notre étude transversale a été conduite en saison sèche pendant laquelle les glossines riveraines vivant en milieux fragmentés, comme celui du foyer de Mandoul, se dispersent peu et restent confinées dans des îlots aux conditions environnementales favorables à leur survie (3). Cette faible dispersion, associée à notre technique d'échantillonnage (rapprochement des pièges dans les sites de capture), justifierait l'absence de glossines capturées en aval de la rivière et probablement la faible densité globale de glossines capturées.

Les Tabanidae en revanche ont été capturées dans les quatre zones prospectées du foyer et en abondance en aval de la rivière. Il est connu que l'activité des Tabanidae varie selon les genres : Chrysops, Haematopota et Atylotus ne sont actifs qu'en saison des pluies, tandis que Tabanus et Ancala ont une activité répartie tout au long de l'année (35). Au Burkina Faso et en Guyane française, il a été observé que les tabanidés, bien que présents toute l'année, ont un pic d'abondance marqué au mois de novembre, en début de la saison sèche $(11,36,37)$. Ces insectes ayant été capturés à des densités très variables, seule une étude longitudinale permettrait de définir les périodes où sont observés des pics de densité dans le foyer de Mandoul. C'est également le cas pour les stomoxes qui n'ont pas été capturés dans la zone 3 et dont l'activité varie en fonction du paysage, du climat (température, humidité, niveau du rayonnement solaire, entre autres) et de l'état physiologique des insectes $(9,27)$.

Les espèces de Tabanidae et de Stomoxyinae capturées dans ce foyer appartenaient aux genres impliqués dans la transmission mécanique de la trypanosomose animale. Cette étude ne permet cependant pas d'établir le degré de leur implication éventuelle dans le cycle épidémiologique de la TAA dont l'endémicité a été montrée. Sachant que les Tabanidae et les Stomoxyinae peuvent transmettre de façon mécanique Trypanosoma sp. $(2,12,29)$, il est toutefois important de dresser le schéma épidémiologique de la transmission de la trypanosomose dans le foyer de Mandoul où circulent à la fois des trypanosomes animaux et le trypanosome humain, T. b. gambiense. 
Les foyers d'endémie mixte THA/TAA sont de nos jours rarement documentés dans l'aire de distribution des glossines. Avec une prévalence minimale de 22 p. 100 estimée par PCR, cette étude princeps sur la trypanosomose animale dans ce foyer montre la prévalence de cette maladie dont la forme due à l'espèce $T$. vivax serait dominante. L'identification de T. brucei s.l. chez des bovins ne confirme pas l'existence de la sous-espèce T. brucei brucei responsable de la TAA. Les amorces utilisées, TBR1/TBR2, spécifiques du groupe des Trypanozoon, donnent également des résultats positifs lors d'une infection de surra due à $T$. evansi $(1,15)$. Par ailleurs, sachant que certains animaux domestiques comme les porcs et les petits ruminants sont en mesure d'héberger T. b. gambiense, responsable de la trypanosomiase chez l'homme (33), il est possible que l'infection à T. brucei s.l. observée chez les bovins soit en fait une infection à T. b. gambiense. Auquel cas, les bovins et éventuellement d'autres animaux domestiques présents constitueraient un réservoir de la maladie du sommeil dans ce foyer.

\section{CONCLUSION}

Cette étude confirme l'endémie concomitante de trypanosomiase humaine et de trypanosomose animale dans le foyer de Mandoul. Concernant la TAA, les rôles respectifs des glossines et des vecteurs mécaniques demandent à être précisés. Au regard de l'importance de l'interface homme-animal-vecteur-écosystème dans le maintien et l'évolution de la trypanosomose dans ce foyer, il paraît impératif que soit clarifié un potentiel rôle du réservoir animal dans la persistance de la THA. Une stratégie d'élimination globale sur la base du concept « One Health » (34) pourrait alors être élaborée afin d'assurer un contrôle durable de l'endémie dans ce foyer.

\section{Remerciements}

Les auteurs expriment leur gratitude aux autorités administratives des cantons de Bodo et Beboto ainsi qu'aux populations des villages composant le foyer de Mandoul.

\section{BIBLIOGRAPHIE}

1. ARTAMA W.T., AGEY M.W., DONELSON J.E., 1992. DNA comparisons of Trypanosoma evansi (Indonesia) and Trypanosoma brucei spp. Parasitology, 1: 67-74.

2. BALDACCHINO F., MUENWORN V., DESQUESNES M., DESOLI F., CHAROENVIRIYAPHAP T., DUVALLET G., 2013. Transmission of pathogens by Stomoxys flies (Diptera, Muscidae): a review. Parasite, 20. DOI: 10.1051/parasite/2013026

3. BOUYER J., 2009. La dispersion des glossines. Insectes, 21.

4. BRUNHES J., CUISANCE D., GEOFFROY B., HERVY J.P., 1998. LeS glossines ou mouches tsé-tsé. Logiciel d'identification et d'enseignement. Montpellier, France, Orstom.

5. BUREAU P., 1996. Historique et évolution de la maladie du sommeil au Tchad. Bull. Liais. Doc. OCEAC, 29 : 90-98.

6. CATTAND P., 2001. L'épidémiologie de la trypanosomiase humaine Africaine : une histoire multifactorielle complexe. Med. Trop., 61 : 313322

7. CHALLIER A., LAVEISSIERE C., 1973 Un nouveau piège pour la capture des glossines (Glossina : Diptera, Muscidae), description et essais sur le terrain. Cah. Orstom Sér. Entomol. Méd. Parasitol., 9 : 251-262.

8. CHAPPUIS F., STIVANELLO E., ADAMS K., KIDANE S., PITTET A., BOVIER A.P., 2004. Card agglutination test for trypanosomiasis (CATT) end-dilution titer and cerebrospinal fluid cell count as predictors of human African trypanosomiasis (Trypanosoma brucei gambiense) among serologically suspected individuals in Southern Sudan. Am. J. Trop. Med. Hyg., 71: 313-317.
9. CHARLWOOD J.D., LOPES J., 1980. Age structure and biting behaviour of Stomoxys calcitrans (L.) (Diptera: Muscidae) from Manaus, Brazil. Bull. Entomol. Res., 70: 549-555.

10. CUISANCE D., 1995 Réactualisation de la situation des tsé-tsé et des trypanosomoses au Tchad. Montpellier, France, Cirad-EMVT, 161 p.

11. DESQUESNES M., DIA M.L., ACAPOVI G., YONI W., 2005. Les vecteurs mécaniques des trypanosomoses animales : généralités, morphologie, biologie, impacts et contrôle. Identification des espèces les plus abondantes en Afrique de l'Ouest. Bobo Dioulasso, Burkina Faso, Cirdes, $67 \mathrm{p}$.

12. DESQUESNES M., DIA M. L., 2003. Trypanosoma vivax: Mechanical transmission in cattle by one of the most common African tabanids, Atylotus agrestis. Exp. Parasitol., 103: 35-43.

13. GARROS C., GILLES J., DUVALLET G., 2004. Un nouveau caractère morphologique pour distinguer Stomoxys calcitrans et S. niger (Diptera : Muscidae). Comparaison des populations de l'île de La Réunion. Parasite, 11 : 329-332.

14. GRUVEL J., 1966. Tse-tse flies as vectors of trypanosomiasis in Chad. Rev. Elev. Méd. Vét. Pays Trop., 19: 169-212. [in French with English abstract]

15. HABILA N., AGBAII A.S., LADAN Z., BELLO I.A., HARUNA E. DAKARE M.A., ATOLAGBE T.O., 2010. Evaluation of in vitro activity of essential oils against Trypanosoma brucei brucei and Trypanosoma evansi. J. Parasitol. Res. DOI:10.1155/2010/534601

16. HASSANE MAHAMAT H., 2003. Enquête épidémiologique et entomologique de la trypanosomiase et son vecteur dans le bassin du Mandoul. N'Djamena, Tchad, Laboratoire de recherche vétérinaire et zootechnique, $11 \mathrm{p}$.

17. JORDAN A.M., 1965. Observations on the ecology of G. morsitans submorsitans in the northern Guinea savanna of northern Nigeria. Bull. Entomol. Res., 56: 1-17.

18. KENNEDY P.G.E., 2013. Clinical features, diagnosis, and treatment of human African trypanosomiasis (sleeping sickness). Lancet Neurol., 12 186-194.

19. KOHAGNE TONGUE L., DIARRA A., PEKA MALLAYE, LOUIS F.J., 2009. Rapport coût-efficacité d'une modification simple de la stratégie diagnostique usuelle de la trypanosomiase humaine africaine. Sci. Med. Afr., 1 : 124-128.

20. LAVEISSIERE C., GREBAUT P., 1990. Recherche sur les pièges à glossines (Diptera : Glossinidae). Mise au point d'un modèle économique : le piège «Vavoua ». Trop. Med. Parasitol., 41 : 185-192.

21. LAVEISSIERE C., HERVOUET J.P., COURET D., 1986. Localisation et fréquence du contact homme/glossine en secteur forestier de Côte d'ivoire. 2. Le facteur humain et la transmission de la trypanosomiase. Cah. Orstom Sér. Entomol. Méd. Parasitol., 25 : 45-57.

22. LOUIS F.J., DJIMADOUM NGAROROUM A., KOHAGNE TONGUE L., SIMARRO P.P., 2008. Le foyer de trypanosomiase humaine africaine du Mandoul au Tchad : de l'évaluation au contrôle. Méd. Trop., 69 : 7-12.

23. LOUIS F.J., KOHAGNE TONGUE L., EBO'O EYENGA V., SIMARRO P.P., 2008. Organisation d'une campagne de dépistage actif de la trypanosomiase humaine africaine à Trypanosoma brucei gambiense. Méd. Trop., 68 : 11-16.

24. MAGNUS E., VERVOORT T., VAN MEIRVENNE N., 1978. A card agglutination test with stained trypanosomes (C.A.T.T.) for the serological diagnosis of T. b. gambiense trypanosomiasis. Ann. Soc. Belg. Med. Trop., 58: $169-176$.

25. MAGRIN G., 2001. Le sud du Tchad en mutation : des champs de coton aux sirènes de l'or noir. Montpellier, France, Cirad, $427 \mathrm{p}$.

26. MASIGA D.K., SMYTH A.J., AYES P., BROMIDGE T.J., GIBSON W.C., 1992. Sensitive detection of trypanosomes in tsetse flies by DNA amplification. Int. J. Parasitol., 22: 909-918.

27. MAVOUNGOU J.F., JAY-ROBERT P., GILLES J., ATSAME EDDA A., DUVALLET G., 2008. Ecologie des stomoxes (Diptera : Muscidae) au Gabon. I. Premier inventaire dans différentes zones écologiques. Parasite, $15: 27-34$

28. MIHOK S., 2002. The development of a multipurpose trap (the Nzi) for tsetse and other biting flies. Bull. Ent. Res., 92: 385-403. 
29. MOHAMMED Y.O., MOHAMED-AHMED M.M., LUBNA T.K. RAYAH I.E. El., 2010. Detection of Trypanosoma brucei gambiense and T. b. rhodesiense in Glossina fuscipes fuscipes (Diptera: Glossinidae) and Stomoxys flies using the polymerase chain reaction (PCR) technique in southern Sudan. Afr. J. Biotech., 9: 6408-6412.

30. MOSER D.R., COOK G.A., OCHS D.E., BAILEY C.P., MCKANE M.R., DONELSON J.E., 1989. Detection of Trypanosoma congolense and Trvpanosoma brucei subspecies by DNA amplification using the polymerase chain reaction. Parasitology, 99: 57-66.

31. NDELEDJE N., BOUYER J., STACHURSKI F., GRIMAUD P., BELEM A.M.G., MBAINDINGATOLOUM F.M., BENGALY Z., ALFAROUKH O.I., CECCHI G., LANCELOT R., 2013. Treating cattle to protect people? Impact of footbath insecticide treatment on tsetse density in Chad. PLoS ONE, 8. DOI:10.1371/journal.pone.0067580

32. NEBOUT M., 1969. Situation épidémiologique de la trypanosomiase au Tchad. Med. Trop., 29: 229-241.

33. NJITCHOUANG G.R., NIIOKOU F., NANA DJEUNGA H.C. MOUNDIPA FEWOU P., ASONGANYI T., CUNY G., SIMO G., 2010. Analysis of the domestic animal reservoir at a microgeographical scale, the Fontem sleeping sickness focus (South-West Cameroon). J. Cell. Anim. Biol., 4: 73-80.

34. OKELLO A.L., BARDOSH K., SMITH J., WELBURN S.C., 2014. One health: past successes and future challenges in three African contexts. PLoS Negl. Trop. Dis., 8: e2884. DOI:10.1371/journal.pntd.0002884

\section{Summary}

Peka Mallaye, Kohagne Tongué L., Ndeledje N., Louis F.J., Mahamat Hassane $\mathbf{H}$. Concomitant transmission of human and animal trypanosomoses: Mandoul focus in Chad

Trypanosomosis is a vector-borne disease which affects both humans and animals. It is cyclically transmitted by tsetse flies and is caused by Trypanosoma sp. Although the disease is mainly endemic where its vectors are present, endemic areas where transmission of both forms of the disease coexist have seldom been studied. During our study, epidemiological and entomological surveys were carried out, followed by the analysis of collected samples by the polymerase chain reaction (PCR). Out of 13,410 persons examined, 132 sleeping sickness cases were diagnosed. After examination of 144 cattle samples by PCR, 33 were found infected by either Trypanosoma brucei (39\%) or T. vivax (55\%), or exhibited a co-infection (two animals). Three insect families were trapped at variable densities (Glossinidae, Stomoxyinae, and Tabanidae). Glossina fuscipes fuscipes was only caught in the southern part of the focus, and the highest apparent density per trap per day (ADT) of 0.56 was found in the gallery forest bordering the villages where the highest number of human African trypanosomosis was diagnosed. Tabanids were caught in all investigated areas but the highest ADT, i.e. 15.55, was observed in the northern part of the focus. No Stomoxys sp. was found in the prospected area located farthest from the river. The identification of trypanosomes in people and in cattle, and the presence of the cyclical vector as well as mechanical vectors, confirmed endemic human and animal trypanosomoses in the focus. Combatting vectors with a strategy common to both forms of the disease is crucial to control them sustainably.

Keywords: Human disease - Animal disease - African trypanosomiasis - Glossinidae - Stomoxyinae - Tabanidae Epidemics - Chad.
35. OLDROYD H., 1973. Tabanidae in Smith (K.G.V.): Insects and other arthropods of medical importance. London, UK, British Museum (Natural History), p. 195-202.

36. OVAZZA M., RICKENBACH A., VALADE M., 1959. Tabanides de la région de Bobo-Dioulasso (Haute-Volta). Répartition et rythme annuel : quelques notes de systématique. Bull. Soc. Pathol. Exot., 52 : 679-698.

37. RAYMOND H.L., 1988. Abondance relative et dynamique saisonnière de Tabanidae (Diptera) d'une savane de Guyane française. Nat. Can., $115: 251-259$.

38. REID R., KRUSKA R.L., DEITCHMANN U., THOTTHON P.K., LEAK S.G.A., 2000. Human population growth and the extinction of the tsetse fly. Agri. Ecosyst. Env., 77: 227-236.

39. SIMARRO P.P., FRANCO J.R., DIARRA A., POSTIGO RUIZ J.A., JANNIN J., 2013. Diversity of human African trypanosomiasis epidemiological settings requires fine-tuning control strategies to facilitate disease elimination. Res. Rep. Trop. Med., 4: 1-6.

40. SIMARRO P.P., LOUIS F.J., JANNIN J., 2003. Sleeping sickness, the forgotten disease: The consequences in the field and a proposal for the action. In: Proc. 27th ISCTRC Conf., Pretoria, South Africa, 29 Sept. - 4 Oct., 2003.

41. SINSHAW A., ABEBE G., DESQUESNES M., YONI W., 2006. Biting flies and Trypanosoma vivax infection in three highland districts bordering Lake Tana, Ethiopia. Vet. Parasitol., 142: 35-46.

Accepté le 28.11.2014

\section{Resumen}

Peka Mallaye, Kohagne Tongué L., Ndeledje N., Louis F.J., Mahamat Hassane H. Transmisión concomitante de tripanosomosis humana y animal: el foco de Mandoul, Chad

La tripanosomosis es una enfermedad que afecta a la vez al hombre y a los animales. Es provocada por Trypanosoma sp. y es cíclicamente transmitida por un vector, la glosina. Aunque esta enfermedad sea esencialmente endémica en el área de distribución de su vector, las zonas endémicas que presentan una transmisión activa de dos tipos de la enfermedad han sido rara vez descritas. En el curso del presente estudio, se llevaron a cabo encuestas epidemiológicas y entomológicas, las muestras obtenidas fueron luego analizadas en el laboratorio mediante la técnica de amplificación en cadena de la polimerasa (PCR). En total, 13410 personas fueron examinadas y 132 casos diagnosticados. El examen de 144 bovinos mediante PCR reveló la infección en 33 de ellos, sea por Trypanosoma brucei (39\%), sea por T. vivax (55\%), sea por una co infección (dos animales). Se capturaron tres familias de insectos (Glossinidae, Stomoxyinae et Tabanidae), en densidades variables. Glossina fuscipes fuscipes fue capturada únicamente en la parte sur del foco y la mayor densidad aparente (DAP $=0,56$ glosinas/trampa/ día) fue observada en la galería forestal bordeando los pueblos en los que fue diagnosticado el mayor número de enfermos. Los Tabanidae estuvieron presentes en todas las zonas estudiadas, pero la mayor densidad (DAP $=15,55$ tabánidos/trampa/día) fue observada en la parte norte del foco. Los estomoxis estuvieron ausentes en la zona estudiada más alejada del río. La identificación de los tripanosomas en el hombre y el ganado y la presencia del vector cíclico y de los vectores mecánicos potenciales confirman la endemia de tripanosomosis animal y humana en este foco. Solo una estrategia global de eliminación permitirá controlarla duraderamente.

Palabras clave: Enfermedad humana - Enfermedad de los animales - Tripanosomosis africana - Glossinidae Stomoxyinae - Tabanidae - Epidemia - Chad. 